\title{
The effect of training trials on passive avoidance deficits in the hippocampectomized rat
}

\author{
ROBERT L. ISAACSON, DAVID S. OLTON, BARBARA BAUER AND PAMELA SWART \\ UNIVERSITY OF MICHIGAN
}

The ability to withhold a response on the basis of a punishing electrical shock after 0, 20, 40 training trials was investigated in rats following radical bilateral hippocampal destruction, damage to the posterolateral neocortex, or sham operations. Hippocampectomized animals continued to make the response despite the previous shock under all training conditions (impaired passive avoidance behavior) but their response latencies were shorter when 20 or 40 training trials had been given.

The extent to which prior acquisition training affects the deficit in passive avoidance behavior in hippocampectomized rats has been recently investigated by Kimble, Kirkby, \& Stein (1966). In their study hippocampectomized animals, as well as control Ss, were trained in two kinds of passive avoidance tasks. The first was one in which no training was given the animals before punishment. Animals received shocks after every exit from a perch located 4-1/2 ft. off the floor. There was no suggestion that the animals with hippocampal destruction left the perch more rapidly than the control groups. On the basis of this latency measure, it was concluded that the limbic lesions produced no significant effect on this passive avoidance task which required no training to establish.

In a second task reported by Kimble et al, the animals were shocked in a goal box located at the end of a runway on the fourth day of training. Normal training, without shock, was continued after the shock trials. In this situation impaired passive avoidance behavior in the hippocampectomized animals was found as expected (e.g., Isaacson \& Wickelgren, 1962; Kimble, 1963; Snyder \& Isaacson, 1965). The significant difference between the two situations was thought to be the number of training trials the animals received in the second task.

The present experiment is also based on manipulation of the training variable, but the results appear to make revision of the interpretation made by Kimble, Kirkby, and Stein seem appropriate.

\section{Procedure}

The experiment utilized a response which would be easily performed by animals, with or without training. The apparatus consisted of a 2 in. wide runway $4 \mathrm{ft}$. above the floor and a small stable cage located about 2 in. from one end of the runway. A $1 / 4 \mathrm{hp}$ motor was attached to a leg of the runway to produce a slight "quiver." The combination of quiver and height was effective in motivating animals to jump the small gap from the runway to the stable cage.
Three groups of albino rats were used. Groups received 0,20 , or 40 acquisition trials before shock. In each training group there were three types of Ss: animals with damage to the hippocampus and posterolateral cortex; animals with only the posterolateral cortex destroyed, and sham operate animals. Ten trials were given each day, with a $30 \mathrm{sec}$. intertrial interval. A total of $53 \mathrm{Ss}$ was used and no group contained less than five Ss. When the specified training period was completed, the Ss were given electrical foot-shock (.8 ma) upon entry into the cage. The animals were then tested for 20 more trials. Each minute of failing to leave the runway was counted as a trial.

\section{Surgery and Histology}

The neocortical and hippocampal destruction was accomplished by aspiration using visual exposure. The operations were performed while the Ss were under barbiturate anesthesia under clean surgical conditions. In making the hippocampal lesions, the neocortical tissue overlying the hippocampus was exposed through a cranial defect and subsequently removed. The lateral ventricles were entered and the hippocampus removed as completely as possible, commensurate with attempts to avoid damage to other subcortical areas. The animals with posterolateral neocortical destruction had the hippocampus exposed but not removed. After the experiment, Ss were sacrificed and perfused with saline and formaline. Paraffin sections were cut at $15 \mu$ and stained after mounting with thionin. Examination of the brains revealed that the destruction in both neocortical and hippocampal ablates was similar to that reported in Isaacson \& Wickelgren (1962) as well as the other studies referenced in this paper.

\section{Results and Discussion}

The results were analyzed in two ways: (1) the mean latency of jumps from the elevated runway to the small cage and (2) the average number of postshock jumps to the cage made in the $60 \mathrm{sec}$. interval. The latency data are presented in Fig. 1. The animals with hippocampal destruction returned to the stable cage more rapidly than the controls even after 0 training trials, although the hippocampectomized animals returned more rapidly after 20 or 40 training trials. Thus, there is partial confirmation of the Kimble et a1, results in that longer latencies, representing diminished failure of passive avoidance, are observed after the no training trial condition relative to the other conditions.

After 40 training trials, the shocks produce markedly distinct differences between the hippocampectomized 


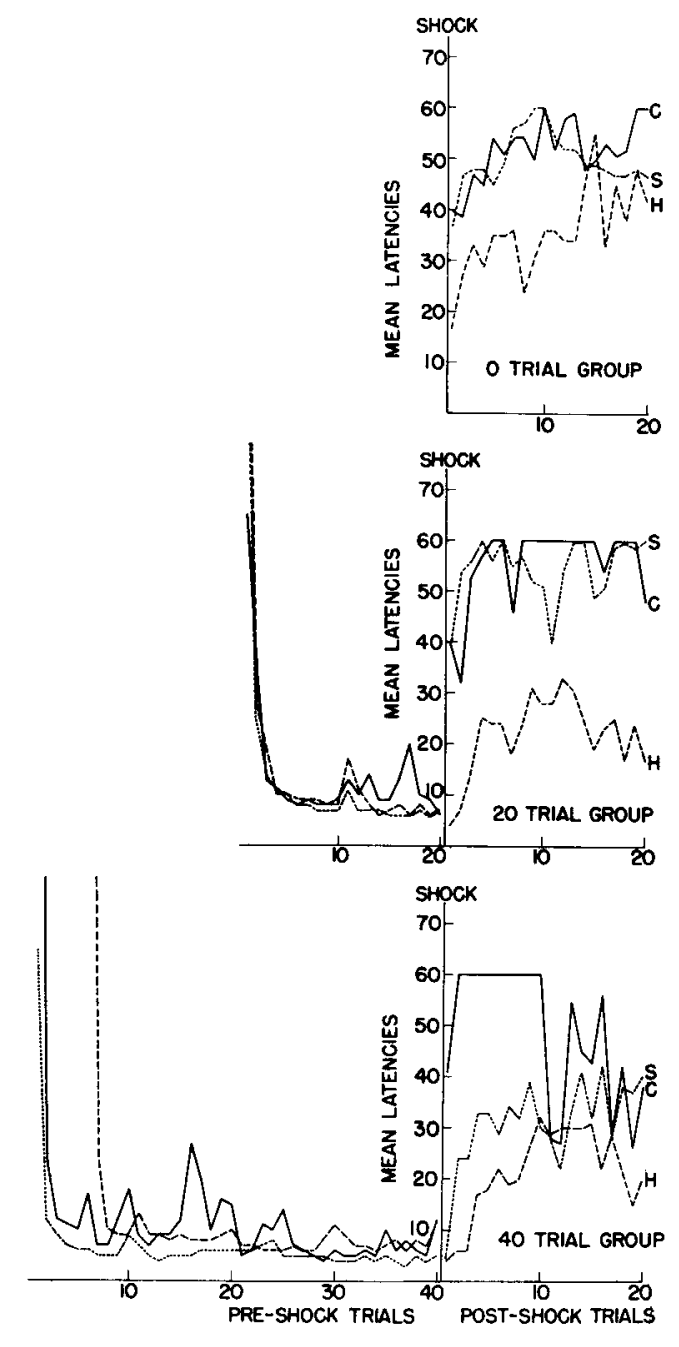

Fig. 1. The mean latencies of response made by the three types of animals in the 20 postshock trials in the three training groups. (S: sham operate animals; H: hippocampal ablates; $C$ : control Ss with destruction of posterolateral neocortex.)

animals and the animals with only neocortical destruction, but a peculiar result is obtained from the sham operate animals. They are intermediate to the lesioned groups and suggest that either the sample of control animals was very peculiar or that the neocortical lesions produced superior levels of passive avoidance behavior under the present conditions of training.

In terms of the mean number of entries into the stable cage, the hippocampally lesioned group is remarkably consistant across all training periods, i.e., means of $13.2,16.0$, and 14.5 over the 0,20 , and 40 training trial conditions. The neocortically damaged and sham operate control animals are similar to each other in the 0 and 20 training trial conditions (means of 5.1 and 5.6 in the 0 trial condition and 3.1 and 4.0 after 20 trials) but diverge in the 40 training trial condition (means of 7.2 and 12.2).

These data, then, present partial confirmation of the Kimble, Kirkby, and Stein results in that the expression of passive avoidance impairment is enhanced when training trials have been given, if a latency measure is used. However, a debility in passive avoidance occurs even without prior acquisition training in terms of entries into the cage after shock.

Differences in procedures may account for the differences between these data and those of Kimble et al. The most significant difference may be that the animals received shocks on all responses when leaving the perch in the Kimble experiment. However, a small number of animals had been run previously in the apparatus of this experiment with the floor of the small cage electrified on all trials after the appropriate number of training trials and results similar to those reported here were found. Perhaps smaller differences in procedures could be vital, including such factors as shock parameters and especially intertrial intervals.

The evidence that impaired passive avoidance can be exhibited without specific, prior training indicates that it is not the training per se which is essential to the passive avoidance deficiency found in the hippocampectomized rats. Perhaps the total behavioral tendency toward the response is the essential factor, however, and this total tendency could have components from prior training and from motivational circumstances as well. Thus, the quivering, shaking, elevated runway may contribute more toward this total response tendency than only the height of the perch used by Kimble et al, and the obtained discrepancies between the studies would then be expected. This could have implications for further studies, such as altering deprivation schedules, in that the passive avoidance impairment would be subject to parametric manipulation, at least in certain tasks.

\section{References}

Isaacson, R. L., \& Wickelgren, W. C. Hippocampal ablation and passive avoidance. Science, 1962, 138, 1104-1106.

Kimble, D. P. The effects of bilateral hippocampal lesions in rats. J. comp. physiol. Psychol., 1963, 56, 273-283.

Kimble, D. P., Kirkby, R. J., \& Stein, D.. G. Response perseveration of passive avoidance deficits in hippocampectomized rats. J. comp. physiol. Psychol., 1966, 61, 141-143.

Snyder, D. R., \& Isaacson, R. L. Effects of large and small bilateral hippocampal lesions on two types of passive-avoidance responses.. Psychol. Rep., 1965, 16, 1277-1290.

\section{Note}

1. This research was supported in part by a grant from the Surgeon General, U.S. Army (DA-MD-49-193-64-G120). 